Meta

Journal des traducteurs

Translators' Journal

\title{
La nature des choses
}

\section{Jean-Paul de Grandpré}

Volume 14, numéro 3, septembre 1969

URI : https://id.erudit.org/iderudit/002502ar

DOI : https://doi.org/10.7202/002502ar

Aller au sommaire du numéro

Éditeur(s)

Les Presses de l'Université de Montréal

ISSN

0026-0452 (imprimé)

1492-1421 (numérique)

Découvrir la revue

Citer cet article

de Grandpré, J.-P. (1969). La nature des choses. Meta, 14(3), 154-158.

https://doi.org/10.7202/002502ar

Ce document est protégé par la loi sur le droit d'auteur. L'utilisation des services d'Érudit (y compris la reproduction) est assujettie à sa politique d'utilisation que vous pouvez consulter en ligne.

https://apropos.erudit.org/fr/usagers/politique-dutilisation/
Cet article est diffusé et préservé par Érudit.

Érudit est un consortium interuniversitaire sans but lucratif composé de l'Université de Montréal, l'Université Laval et l'Université du Québec à Montréal. Il a pour mission la promotion et la valorisation de la recherche. https://www.erudit.org/fr/ 


\section{PROBLÈMES ET SOLUTIONS}

\section{LA NATURE DES CHOSES}

La qualité du français traduit, au Canada, semble s'améliorer. Il est bon de dire sa conviction qu'il en est ainsi. Un bilan des dix dernières années s'avérerait probablement positif. Le nombre des traducteurs, à l'aise dans leur discipline et clairs dans le propos, est, en tout cas, en nette augmentation.

Un optimiste se réjouirait de ces modestes victoires. Mais gardons-nous de céder naïvement à un pareil sentiment. Il pourrait trop facilement servir à masquer chez nous le problème de la langue, lequel n'a rien perdu de sa gravité. Sans doute y a-t-il actuellement prolifération de vocabulaires et de lexiques de toutes sortes. Mais combien méritent d'être retenus ? Combien vont au fond des choses ? Que de facettes du barbarisme pourchassé restent dans l'ombre! Que de provignements, que d'acceptions connexes sont négligés! Si bien que plusieurs de ces travaux, même s'ils sont méritoires, risquent d'être un cautère sur une jambe de bois.

Il n'est pas question de lancer l'anathème à quiconque. Nous sommes hélas ! tous trop vulnérables en matière de traduction pour porter des accusations péremptoires. D'ailleurs, les excuses valables ne manquent pas. Les techniques évoluent rapidement et quel praticien, par exemple, qui, croyant connaitre le fort et le faible de son métier, ne s'est pas vu soudain sous les feux croisés d'une mitraillade de termes entièrement nouveaux.

Il y a aussi la guérilla des vocables familiers. Simples en apparence, ils recouvrent souvent des réalités complexes, des nuances insoupçonnées. J'en donnerai un exemple. On considère très généralement au Canada que l'aire sémantique du mot anglais employee se juxtapose exactement sur celui de son homonyme français, employé. Il n'en est rien. Le premier en effet se définit comme " a person working for another person or a business firm for pay ${ }^{1}$. Le registre du second est beaucoup plus restreint: il sert à désigner les personnes travaillant dans l'administration, les bureaux, et aussi les commis de magasins ${ }^{2}$. La différence est importante. Elle

1. The American College Dictionary, New York, Random House, 1956.

2. Cf. Pierre Daviault, Langage et traduction, Ottawa, Bureau fédéral de la traduction, Secrétariat d'Etat, 1963. 
est aussi révélatrice d'un état de choses inquiétant, si l'on songe que des glissements de ce genre fourmillent dans notre parler, et ce qui est pis, passent inaperçus.

Voici un certain nombre de citations rendant compte de l'acception propre et des résonances sociales du mot employé, et par voie de conséquence, des harmoniques sémantiques de son doublet employee. Elles sont toutes tirées de l'Express.

«La cité [Paris] devient le lieu de résidence privilégiée des classes les plus favorisées: cadres, patrons et employés. ${ }^{3}$ «Il n'est pas possible d'en faire [de Paris] une capitale de fonctionnaires ou d'employés, un Washington à la française. $\gg^{4}$ D'où l'on voit l'aspect légèrement prestigieux de employé, qui peut dans certains cas, en terminologie administrative, s'élever au niveau de officer.

«C'est aussi cette prise de conscience des employés dont les ouvriers défenestraient, il y a dix ans, les machines à écrire. ${ }^{5}$ Nous voyons ici que les ouvriers dans leur colère ont assimilé les employés aux patrons. Il y a affirmation d'une catégorie de travailleurs par rapport à une autre. Donc lutte des classes. Lorsqu'ils conjugueront leurs efforts contre leurs dirigeants, employés et ouvriers seront désignés par un autre vocable qui, changeant l'éclairage, ne nous fixera pas moins sur la composition du groupe.

«On ne sait plus si c'est l'austérité... qui provoque les revendications des salariés, ou si ce sont celles-ci qui conduisent à l'austérité. " ${ }^{6}$ "Le mouvement qui a paralysé la totalité des 23 usines Ford et de leurs 4800 salariés est parti de la base, et non des instances syndicales. ${ }^{7}$ Salarié englobe donc en une même expression employés et ouvriers. Il sert aussi, d'autre part, à opposer ceux qui touchent un salaire fixe (ouvriers, employés et cadres) aux travailleurs indépendants (professions libérales, commerçants et autres). Pourtant un chef d'entreprise soucieux de concision a besoin d'autres noms génériques. Là encore, les rédacteurs de l'Express viendront à la rescousse.

«Un contrat d'entreprise signé entre la direction et les représentants du personnel vient à échéance le 31 mars. $»^{s}$ *Le nouveau contrat d'entreprise confirme et accrôt les avantages que les collaborateurs du journal tenaient du précédent contrat. ${ }^{9}$ 《 La contribution de l'entreprise variera suivant le niveau de salaires de manière à favoriser les titulaires de revenus modestes pour qui l'acte d'épargne est plus difficile. $\gg 10$

La distinction à faire entre employé et employee se présente sous un angle différent en assurance sur la vie. Cette industrie pratique des régimes de prévoyance ${ }^{11}$ à l'intention des personnels d'entreprises. Il arrive même que le groupe assuré soit une simple association de membres n'ayant pas un statut de travailleur: des Chevaliers de Colomb, par exemple. Là encore, il faut, par souci de concision, désigner ces personnes par une seule expression. L'anglais, n'a pas réussi à le faire de façon commode. Le français, très à l'aise sur le plan abstrait et apte à la

3. L'Express, n ${ }^{\circ} 870$, p. 5.

4. Ibid., p. 6 .

5. Ibid., n $\mathrm{n}^{\circ} 828$, p. 13.

6. lbid., $\mathrm{n}^{\circ} 924$, p. 55

7. Ibid.

8. Ibid., p. 24

9. Ibid., p. 25 .

10. Ibid.

11. Ces contrats peuvent aussi bien s'appeler régime de pension ou caisse de retraite. 
généralisation, appelle le souscripteur membre adhérent et les assurés de toutes catégories membres participants. Voilà qui est commode, clair et concis.

Autres expressions propres à ces contrats: employees' contributions, cotisations des participants ou cotisations salariales, selon le cas; employer's contributions, contributions des adhérents ou contributions patronales, le cas échéant; employee pension fund, régime de prévoyance d'entreprise ou régime de prévoyance de personnel.

Dernière observation, les membres des syndicats, quelle que soit leur profession, sont des adhérents: "Les adhérents commencent à douter de l'action syndicale. Ils ne voient plus où elle débouche. ${ }^{12}$

Le court exposé qui précède répond à deux fins. Il sert d'abord à faire apparaître la difficulté propre à la fabrication des vocabulaires bilingues. Peut-être aura-t-on entrevu que le choix des équivalences sémantiques s'inscrit dans la nature même des êtres et des choses. L'écart entre les réalités façonnées de part et d'autre par des civilisations différentes, nécessite souvent en langue d'arrivée une restructuration des terminologies, à tout le moins un refus a priori des parallélismes faciles. Ce travail doit s'effectuer en fonction de la connaissance des vocables à transposer, des dynamismes propres aux nations en présence et des ressources psychologiques des langues en contact. Ensuite et de façon plus générale, l'exposé en question a pour but de poser le problème de la traduction en terre canadienne à la lumière des exigences et des répercussions de cette discipline sous le rapport de notre idiome.

Il reste donc, par un élargissement des perspectives, à inférer des difficultés évoquées aux moyens propres à améliorer la situation. Limitant les mesures préconisées à trois domaines particuliers, je m'adresserai successivement à la profession, à l'industrie et aux gouvernements.

Même pourvu d'une solide formation universitaire, c'est-à-dire en possession des principes et de la méthode, le jeune traducteur reste à pied d'œuvre devant la matière à traiter et la terminologie qui lui est propre. Ignorant à quel secteur d'activité il était destiné, il n'a pu, pendant ses études, se donner la spécialisation nécessaire. L'acquisition des nouvelles connaissances, sur le terrain, c'est-à-dire à partir d'une langue dégradée, sera un travail de longue patience. Ce n'est que peu à peu, après des années de réflexion et d'observation, que le substrat des vocables et des réalités lui sera révélé. Tout au long de cet apprentissage, il devra, scrupuleusement et méthodiquement, consigner par écrit ses découvertes. Il pourra ainsi, le moment venu, en assurer la diffusion et en généraliser l'usage.

La lecture de textes sûrs, en langues de départ et d'arrivée, sera pour lui un aliment indispensable. Il devra y consacrer de longs moments. Refuser de le faire serait se condamner à une autarcie malsaine, susceptible de provoquer à brève échéance l'appauvrissement du « métier ».

11 est des tournures, il est des transpositions faisant appel à des principes qui, sans le rapprochement des textes, risquent de demeurer inappliqués. Qu'on en juge: «This policy is issued on the basis of the application and in consideration of the payment of premiums», se rend par: "La police est établie sur la foi de la

12. L'Express, no 924, p. 8. 
proposition qui lui sert de base et moyennant le paiement des primes ». Qu'ont de commun ces deux phrases, sinon leur sens et leur caractère idiomatique. Pourquoi, par exemple, emploie-t-on le démonstratif en anglais (this policy) et, en français, l'article ? Pourquoi on the basis fait-il l'objet d'une transposition en deux temps: «sur la foi de » et «qui lui sert de base»? De savantes considérations d'ordre juridique et linguistique peuvent sans doute rendre compte de ces faits de langue. II n'en demeure pas moins qu'une simple lecture consciente en permet l'appréhension.

Tributaire de la communication pour une organisation efficace de la production, l'industrie a également des obligations sur le plan social. Elle est de ce chef tenue de prendre les moyens d'assurer un bon fonctionnement de la langue des points de vue technique et qualitatif. Point n'est besoin d'insister sur ces différents aspects: ils ne peuvent être dissociés; ils sont même complémentaires. Il convient plutôt de s'attacher à la façon d'attirer et de garder dans l'industrie les spécialistes capables d'assumer cette tâche difficile. Les chefs d'entreprise y réussiront pour autant qu'ils sauront offrir à ces sujets de bonnes conditions de travail.

On devra particulièrement surveiller les normes quantitatives. Les traducteurs ne sont pas des duplicateurs. Ils doivent rester seuls juges de la quantité du travail à faire dans les délais impartis. C'est là une condition essentielle à la qualité recherchée. Il faudra aussi leur accorder du temps pour la recherche et au besoin les libérer en leur adjoignant un personnel pour le travail de documentation et l'expédition des besognes courantes.

On aura dès maintenant compris que le traducteur - titulaire d'un grade universitaire, rompu à sa discipline et responsable devant le public et ses commettants du rôle social que ces derniers doivent assumer dans une société bilingue - commande un traitement respectable. Il ne serait nullement exagéré de le situer à mi-chemin, disons, entre celui des cadres intermédiaires et celui des cadres supérieurs.

En ces temps où la prise de conscience linguistique se généralise chez les francophones canadiens, où la profession est de ce fait sollicitée de partout, seuls des salaires alléchants permettront aux entreprises anglophones de remédier durablement à une situation qui les concerne au plus haut point.

S'adresser à nos gouvernements demeure chose délicate. Sans doute n'ignoret-on pas dans ces milieux les failles de notre système linguistique. Des organismes ont été mis en place pour y remédier. Seront-ils de simples palliatifs ? Combien de temps faudra-t-il attendre avant que n'apparaissent des résultats concrets? Quand pourrons-nous enfin sortir de l'expectative? Le traducteur céderait ici volontiers la parole au simple citoyen, mais ce dernier ne pourrait, dans son désarroi, s'empêcher d'interroger le premier.

Est-il bien vrai, comme le dit Galichet que le «langage traduit la représentation qu'une communauté humaine se fait du monde $» 13$ ? Est-il bien vrai, comme l'affirme le même auteur, que la langue est un "instrument de pensée » ${ }^{14}$, donc un mécanisme essentiel à l'activité créatrice et permettant à l'homme d'exprimer

\footnotetext{
13. Georges Galichet, Physiologie de la langue française, Paris, Presses Universitaires de France, "Que sais-je? », 1964, p. 6.

14. Ibid., p. 5 .
} 
sa personnalité ? Ce célèbre grammairien a sans doute raison. La langue, qui permet à l'homme de trouver sa propre dimension psychologique, le met à même; surtout dans une société dualiste comme la nôtre, de dépasser le stade d'une existence anonyme. Elle est le préalable à l'affirmation d'une personnalité autonome par la créativité.

Comment dès lors pourrons-nous, Québécois, Canadiens francophones, nous affirmer individuellement et collectivement? Notre idiome n'est-il pas un parler artisanal (lousy French, a dit M. Trudeau!), une langue contrariée dans son économie, faussée dans sa syntaxe et son lexique. Comment pareilles carences ne diminueraient-elles pas moralement et intellectuellement l'homo quebecencis? Nos prédécesseurs ont été lents à reconnaître l'existence de ce terrible handicap, mais les générations montantes le sentent avec acuité.

Privés par leur indigence verbale de la possibilité de s'exprimer convenablement, certains se réfugient dans le mutisme. D'autres, cédant sous la poussée d'une émotivité traumatisante, choisissent de jargonner au grand jour. D'autres enfin s'installent dans un processus de pensée revendicative. Faisant fi d'une cité où leur intégration est entravée à la base, ils cherchent à s'annexer les puissances du nationalisme par le biais de la colère. Mais leur violence, que nous réprouvons, re serait-elle pas dans la nature des choses?

L'enjeu est capital. Aucun des éléments à considérer ne peut être négligé. Notre situation géographique nous oblige à traduire; notre situation politique, à bien traduire. La bonne traduction est l'un des facteurs les plus importants de la régénérescence de notre idiome. Il en sera ainsi si l'on veut bien prendre les moyens de former d'excellents traducteurs et si l'on consent à leur donner sur les plans social et économique un statut correspondant à l'importance de leur mission.

JeAn-Paul de Grandpré

1. Ont servi de base à cette étude, les définitions fournies par le Grand Larousse encyclepédique, 10 vol., Paris, Larousse, 1960; et le Dictionnaire alphabéthique et analogique de la langue française, 6 vol., par Paul Robert, Paris, Société du Nouveau Littré, 1965. 